\title{
Magnetic Levitation as a Tool for separation: Separating Cocrystals from Crystalline phases of individual compounds
}

\author{
Chloé Matheys,† Natalia Tumanova, † Tom Leyssens, ${ }^{*} †$ and Allan S. Myerson $\ddagger$ \\ † IMCN, MOST, Université Catholique de Louvain, 1, Place Louis Pasteur, B-1348, Louvain-la-Neuve, \\ Belgium. \\ $\ddagger$ Department of Chemical Engineering, Massachusetts Institute of Technology, 77 Massachussets \\ Avenus, Cambridge, MA 02139 .
}

\section{Supporting information}

\section{Materials}

Carbamazepine (CAS 298-46-4; 97\% chemical purity) was purchased from TCI. D(+)-Camphoric acid (CAS 124-83-4; 99\% chemical purity) and D(+)-Sucrose (CAS 57-50-1; 99\% chemical purity) were purchased from Acros Organics. Salicylic acid (CAS 69-72-7 99\%; chemical purity) was purchased from Sigma-Aldrich. Manganese(II)-chloride Tetrahydrate (CAS 13446-34-9 99\% chemical purity) was purchased from Carl Roth. All were used with no further purification.

Tween 20 (Polysorbate 20; CAS 9005-64-5) was purchased from Alfa Aesar and was diluted five times before utilization.

Acetonitrile (HPLC grade), ethanol (absolute), and sodium chloride (CAS 7647-14-5; 99\% chemical purity) were purchased from VWR International and used with no further purification.

\section{Methods}

HPLC

HPLC, or high performance liquid chromatography is an analytical tool that separates, identifies and quantifies (based on calibration measurements) compounds of a solution.

To separate CBZ and SA, we used an Atlantis column T3 C18 $(50 * 4,6 \mathrm{~mm} 3 \mathrm{mic})$, eluent acetonitrile $/ \mathrm{H}_{2} \mathrm{O}$ miliQ, gradient $90 / 10 \mathrm{H}_{3} \mathrm{PO}_{4}$, flow $1 \mathrm{~mL}^{*}$ min-1, retention times: $\mathrm{SA} 2.9$ min CBZ 3.1 min. To separate CBZ and CA, we used a Waters Sunfire column T3 C18 (100*4,6mm 3,5mic), eluent acetonitrile/ $\mathrm{H}_{2} \mathrm{O}$ miliQ, gradient $90 / 10 \mathrm{H}_{3} \mathrm{PO}_{4}$, flow $1 \mathrm{~mL}^{*}$ min-1, retention times: $\mathrm{CA} 4.4 \mathrm{~min}$ CBZ 5.4 $\min$.

\section{XRPD}

X-ray Powder Diffraction (XRPD): X-ray diffraction (XRD) reflectance measurements were performed with a Siemens D5000 80 diffractometer equipped with a $\mathrm{Cu} X$-ray source operating at $40 \mathrm{kV}$ and 40 $\mathrm{mA}$ and a secondary monochromater allowing to select the $\mathrm{K} \alpha$ radiation of $\mathrm{Cu}(\lambda=1.5418 \AA$ ). $\mathrm{A}$ scanning range of $2 \theta$ values from $2^{\circ}$ to $72^{\circ}$ at a scan rate of $0.6^{\circ}$ min-1 was applied. Small amounts of sample were placed on the sample holder and measured at ambient temperature. 


\section{MagLev}

Device

The MagLev device was engineered to be easy, inexpensive and portable. It is composed of two permanent NdFeB magnets with like-pole facing one another and the axis between the poles aligned with the gravitational force. They are separated by a paramagnetic solution.
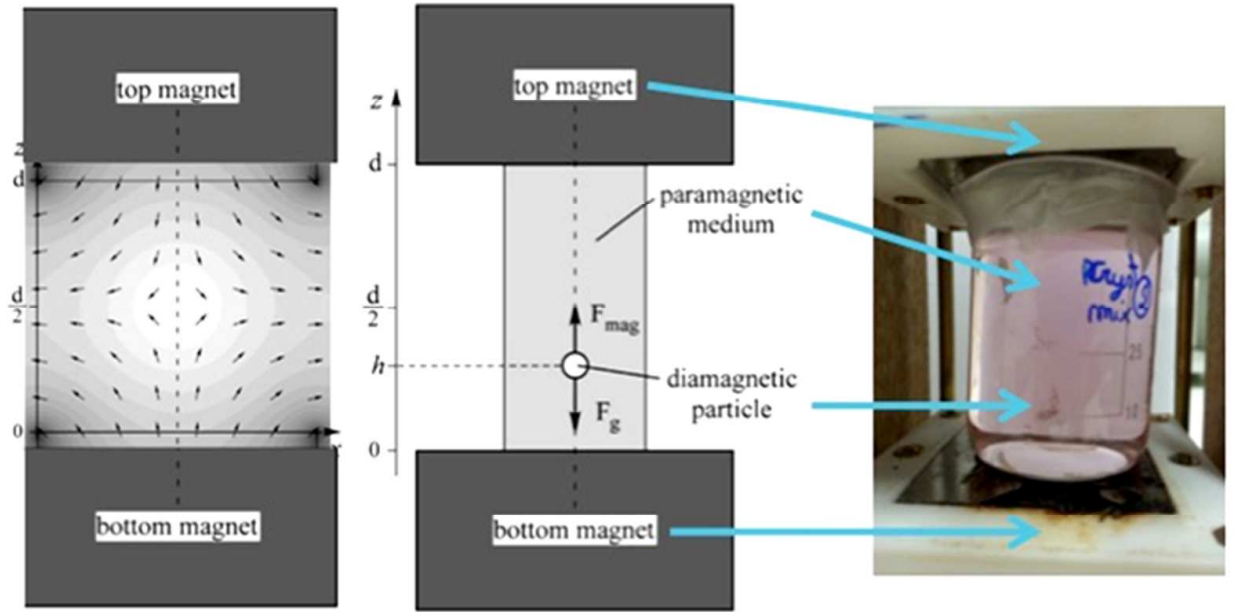

We used $5 * 5 * 2 \mathrm{~cm}$ NdFeB magnets ( $B_{0}$ the magnetic field at the magnet surface $\approx 0,4 \mathrm{~T}$ ) separated by $6 \mathrm{~cm}$. An aqueous solution of $\mathrm{MnCl}_{2}, \mathrm{NaCl}$ and sucrose was used as the paramagnetic solution. The experiments were carried out in a temperature controlled room at $21^{\circ} \mathrm{C}$.

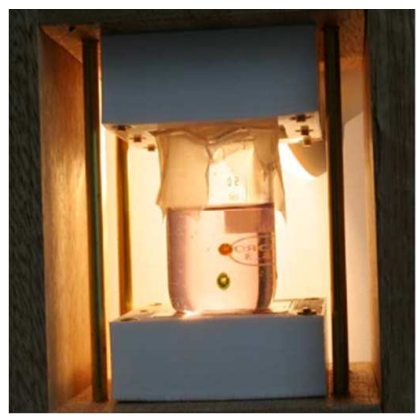

\section{Principle}

The MagLev is based on the equilibrium of a magnetic force and the gravitational force. The magnetic force varies linearly as a function of the height between the magnets while the gravitational force (whose magnitude depends on the density of the particle) and the Archimedes thrust remain constant all along. Compounds will thus find their equilibrium at a different height depending on their densities. G. M. Whitesides' group established a theoretical relation between a compound's density and its height in the MagLev. The derivation of this equation is rather complex, and described in literature.

$$
h=\frac{\left(\rho_{\mathrm{s}}-\rho_{\mathrm{m}}\right) g \mu_{0} d^{2}}{\left(\chi_{\mathrm{s}}-\chi_{\mathrm{m}}\right) 4 B_{0}^{2}}+\frac{d}{2}
$$

We can see that the height is influenced by a large number of variables which are described below, and depends linearly on the density of the suspended object:

$$
\rho_{\mathrm{s}}=\alpha h+\beta
$$

With:

$$
\alpha=\frac{4\left(\chi_{\mathrm{s}}-\chi_{\mathrm{m}}\right) B_{0}^{2}}{g \mu_{0} d^{2}} \quad \beta=\rho_{\mathrm{m}}-\frac{2\left(\chi_{\mathrm{s}}-\chi_{\mathrm{m}}\right) B_{0}^{2}}{g \mu_{0} d}
$$


As the suspended object is diamagnetic (we are working with organic compounds), its magnetic susceptibility will to be lower than the one from the paramagnetic media. $\left(\chi_{s}-\chi_{m}\right)$ will therefore be negative and so will $\alpha$. Given the same reasoning, $\beta$ is positive.

Using this calculus, magnetic levitation can be used as a tool to measure the density of a material with a precision up to $0,0002 \mathrm{~g} / \mathrm{cm}^{3}$. Some limitations will appear with the practical use of the device, which will be described in more detail.

\section{Variables impacting Maglev \\ $t \underline{\rho}_{\mathrm{s}}$ - density of the suspended object}

The density of an object is an intrinsic property. A higher $\rho_{\mathrm{s}}$ will lead to a lower levitation height. When separating two compounds, a higher density difference will lead to a better separation.

$\rho_{m}$ - density of the media

The density of the paramagnetic solution has to be adjusted so it stands in the same range as the suspended object(s) density. If $\rho_{\mathrm{m}}$ is too high, the object floats; preventing all measurement. If it is too low, the object sinks and again no measurement is allowed.

$\rho_{\mathrm{m}}$ of an aqueous $\mathrm{MnCl}_{2}$ solution can be modified by the addition of $\mathrm{NaCl}$ or sucrose. Sucrose is used to increase the viscosity of the solution in such a manner as to prevent thermal motion of particles (Brownian motion) in the solution but nevertheless allowing for some motion as to achieve separation.

$\underline{\mu}_{0}, \underline{B}_{0}, g$ - constants

$\mu_{0}$, the magnetic permeability of free space $\left(4 \pi \times 10^{-7} \mathrm{~N} / \mathrm{A}^{2}\right), \mathrm{B}_{0}$ the magnetic field at the magnet surface $\left(\approx 0,4 \mathrm{~T}\right.$ for $\mathrm{NbFeB}$ magnets) and $\mathrm{g}$, the gravity $\left(9,81 \mathrm{~m} / \mathrm{s}^{2}\right)$ are constants that appear in the equation when calculating the density of an object based on its levitation height.

$\mathrm{d}$-distance between the magnets

The distance between the magnets will impact the strength of the magnetic field within the device. As this distance can be varied, one has to be careful to keep it constant between experiments; we chose to fix it ay $6 \mathrm{~cm}$, the height of a $50 \mathrm{ml}$ beaker.

$\chi_{s}$ - magnetic susceptibility of the suspended object

As all the objects we are going to analyse are organic compounds, they are diamagnetic and their magnetic susceptibility is negative and of the same magnitude. We can thus assume will thus $\chi_{\mathrm{s}}$ doesn't affect the levitation height of the suspended object.

$\chi_{m}$ - magnetic susceptibility of the media

The magnetic susceptibility of an aqueous solution depends on the amount of paramagnetic ions that are dissolved in it. We decided to work with $\mathrm{MnCl}_{2}$. A more paramagnetic solution (containing more $\mathrm{MnCl}_{2}$ ), will lead to the separation of objects with very different densities, while a less paramagnetic one can be used to separate objects with very close densities.

A highly paramagnetic solution is less sensitive, as a lightly paramagnetic solution is more sensitive. Temperature

It is important to note that all those parameters are temperature dependant. The MagLev device has to be used at a controlled temperature. All our experiments were performed in a temperature controlled room at $21^{\circ} \mathrm{C}$.

\section{Limitations}

Solubilisation in water

As we need to introduce the crystals into an aqueous solution of $\mathrm{MnCl}_{2}$ for the separation, the compound should not be soluble in water. Of course, no compound is totally insoluble. The method is in fact limited to the use of compounds whose dissolution rate allows them to get separated before significant solubilisation occurs. 


\section{Particle size}

The MagLev can only separate crystals with a diameter larger than $5 \mu \mathrm{m}$. For smaller particles, Brownian motion becomes non-negligible; the gravitational and magnetic forces are not predominant anymore. There is no well-defined equilibrium height and the particles remain dispersed. In general, the smaller the particles, the larger the cloud of crystals one observes around the central levitation height. This 'clouding' may limit a separation of the several phases of a system as clouds can overlap.

The Maglev is therefore not suitable for materials that were obtained by grinding. For the purification of cocrystal, this is a downside as most cocrystals are obtained through this method.

Separation time

It was shown that there is a link between the size of the particle and the time they require to reach their levitation height. The smaller the particle, the longer it takes to reach the equilibrium height. The time can vary from one hour to three days. In our case, we no longer observed changes after 24h.

Particle aggregation

For hydrophobic particles, one can imagine hydrophobic aggregation of crystals. If crystals of different phases and thus different levitation heights are stuck together in the same aggregate, the aggregate will position itself between the two equilibrium heights and no separation would occur. To counter this effect, $0,05 \%$ of Tween 20 (surfactant) can be added to the paramagnetic solution. Air into the medium

As the MagLev device is in contact with ambient air, the paramagnetic solution contains solubilised gases that can stick to the crystal surface and modify their density. To avoid this, a larger amount of surfactant can be added (up to 1\% Tween 20) and the solution degassed in an ultrasound bath.

Air can also intervene in another way; by occurring in inclusions inside the crystals and modifying their density. This cannot be countered by the addition of surfactant or sonication. One should therefore carefully check the quality of the crystalline material one wants to separate.

Density range

When working into $\mathrm{MnCl}_{2}$ water, the density range of compounds to be separated should stand between 1.2 and $1.5 \mathrm{~g} / \mathrm{cm}^{3}$ in order to levitate into the medium and not sink or float.

\section{Phase Diagram Construction}

Cocrystal ternary phase diagram

A co-crystal ternary phase diagram allows visualizing the different phases one can obtain according to the overall composition of the system and at a given temperature. One typically identifies six different regions in a cocrystal ternary phase diagram :

- $\quad L$ : Solution (everything is dissolved).

- $A_{s}+L$ : Suspension of pure $A$ crystals in a solution containing $A$ and $B$.

- $A_{s}+A: B_{s}+L$ : Mixture of $A$ crystals and $A: B$ cocrystals in suspension. The solution composition is now fixed at the eutectic composition (C1).

- $A: B_{s}+L$ : Pure $A B$ cocrystal in suspension.

- $B_{s}+A: B_{s}+L$ : Mixture of $B$ crystals and $A: B$ cocrystals in suspension. The solution composition is now fixed at the eutectic composition (C2).

- $B_{s}+L$ : Suspension of pure B crystals and a solution containing B and A. 


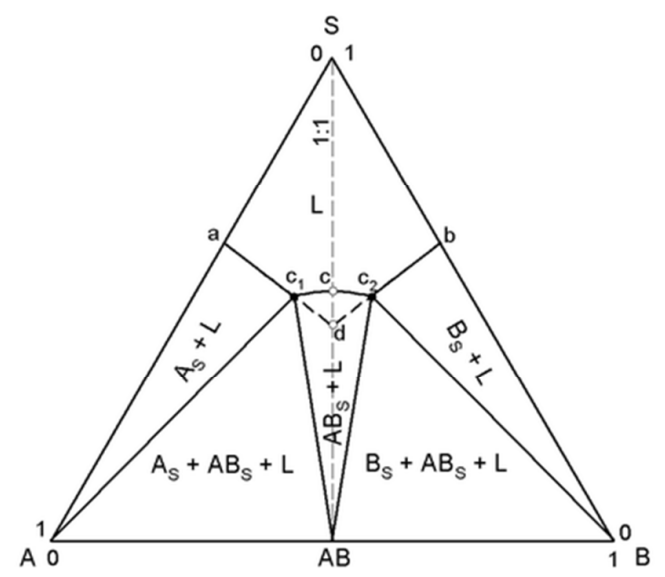

Figure S.1 - Six different regions of a cocrystal ternary phase diagram

A ternary phase diagram can easily be established experimentally. The saturation curve of a two solid phase diagram (delimiting the L region; see fig. 1) represents the concentration of the saturated solutions for every API/coformer ratio.

To establish such a diagram, one simply has to evaluate the composition of several saturated solutions containing different ratios of $\mathrm{API} /$ coformer, as shown in fig. 2.

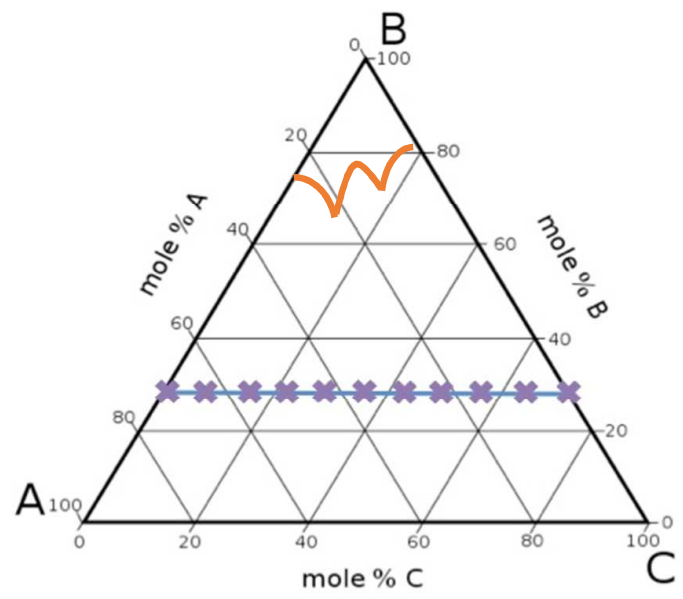

Figure S.2 - Overall compositions of 11 samples used to build a liquidus curve of a phase diagram

Vials are created containing a given amount of solvent, $A$, and $B$, upon which they are heated up to total dissolution. Solutions are cooled, and seeded with all possible solid forms ( $A, B$ and $A B)$. After storing for a minimum of $48 \mathrm{~h}$, vials are seeded once more with all possible solid forms. Supernatant solutions (eg. 100 $\mu \mathrm{l}$ ) are then collected and analyzed using a reverse HPLC and a calibration curve. The exact concentration of CBZ and SA are determined, and the amount of $A C N$ is approximated to $100 \mu \mathrm{l}$. This is a valid approximation as the total amount of ACN is of the order of $95 \%$.

The crystals are also collected and HPLC and XRPD measurements performed.

Thanks to the HPLC measurements and the calibration curve, the concentration of API and coformer in the supernatant solution can be determined. This yields a data point on the liquidus line (API, coformer and solvent. 


\section{$\underline{\mathrm{CBZ} / \mathrm{SA} \text { in } \mathrm{ACN} \text { at } 9^{\circ} \mathrm{C}}$}

Table S.1 Composition of initial vials with different ratios of CBZ and SA.

\begin{tabular}{|c|c|c|c|}
\hline Vial & W CBZ $(\mathrm{mg})$ & $\begin{array}{c}\text { W SA } \\
(\mathrm{mg})\end{array}$ & $\begin{array}{c}\text { Eq SA } \\
\text { start }\end{array}$ \\
\hline 1 & 0 & 88,42 & $/$ \\
\hline 2 & 5,43 & 79,82 & 25,15 \\
\hline 3 & 9,93 & 70,22 & 12,10 \\
\hline 4 & 14,84 & 60,84 & 7,01 \\
\hline 5 & 19,75 & 52,88 & 4,58 \\
\hline 6 & 24,83 & 43,87 & 3,02 \\
\hline 7 & 29,61 & 35,81 & 2,07 \\
\hline 8 & 35,76 & 26,41 & 1,26 \\
\hline 9 & 39,86 & 17,2 & 0,74 \\
\hline 10 & 44,61 & 8,84 & 0,34 \\
\hline 11 & 49,45 & 0 & 0,00 \\
\hline
\end{tabular}

\begin{tabular}{|c|c|}
\hline $\begin{array}{c}\text { Eq SA } \\
\text { crystal }\end{array}$ & $\begin{array}{c}\text { Solid phase } \\
\text { (XRPD) }\end{array}$ \\
\hline$/$ & SA \\
\hline$/$ & SA \\
\hline 34,5 & No Data \\
\hline 1,89 & No Data \\
\hline 0,93 & No Data \\
\hline 0,91 & No Data \\
\hline 0,88 & Cocrystal \\
\hline 0,89 & No Data \\
\hline 0,92 & No Data \\
\hline 0,20 & $\begin{array}{c}\text { CBZ (less } \\
\text { clear data) }\end{array}$ \\
\hline 0,00 & CBZ \\
\hline
\end{tabular}

Table S. 2 Composition of the supernatant solution after thermodynamic equilibrium has been reached.

\begin{tabular}{|c|c|c|c|c||c|c|c||}
\hline \multirow{2}{*}{} & \multicolumn{3}{|c||}{ Saturated solution composition } & \multicolumn{3}{c||}{ Mass percentage } \\
\cline { 2 - 7 } & $\begin{array}{c}\text { C CBz } \\
(\mathrm{mmol} / \mathrm{ml})\end{array}$ & $\begin{array}{c}\mathrm{C} \mathrm{SA} \\
(\mathrm{mmol} / \mathrm{ml})\end{array}$ & $\begin{array}{c}\mathrm{n} \mathrm{AcN} \\
(\mathrm{mmol} / \mathrm{ml})\end{array}$ & Nb éq SA & $\% \mathrm{CBz}$ & $\% \mathrm{SA}$ & $\% \mathrm{AcN}$ \\
\hline 1 & 0,000 & 0,30 & 19,15 & $/$ & 0,00 & 4,94 & 95,06 \\
\hline 2 & 0,015 & 0,26 & 19,15 & 16,94 & 0,43 & 4,30 & 95,26 \\
\hline 3 & 0,031 & 0,37 & 19,15 & 12,05 & 0,86 & 6,11 & 93,02 \\
\hline 4 & 0,028 & 0,33 & 19,15 & 11,83 & 0,79 & 5,50 & 93,71 \\
\hline 5 & 0,038 & 0,30 & 19,15 & 7,95 & 1,06 & 4,99 & 93,94 \\
\hline 6 & 0,036 & 0,25 & 19,15 & 6,84 & 1,02 & 4,10 & 94,87 \\
\hline 7 & 0,035 & 0,17 & 19,15 & 4,96 & 0,99 & 2,90 & 96,09 \\
\hline 8 & 0,071 & 0,11 & 19,15 & 1,60 & 2,03 & 1,92 & 96,02 \\
\hline 9 & 0,099 & 0,06 & 19,15 & 0,62 & 2,78 & 1,03 & 96,12 \\
\hline 10 & 0,156 & 0,06 & 19,15 & 0,41 & 4,27 & 1,06 & 94,52 \\
\hline 11 & 0,164 & 0,00 & 19,15 & 0,00 & 4,53 & 0,00 & 95,29 \\
\hline & & & & & & & \\
\hline
\end{tabular}

a)

Acetonitrile

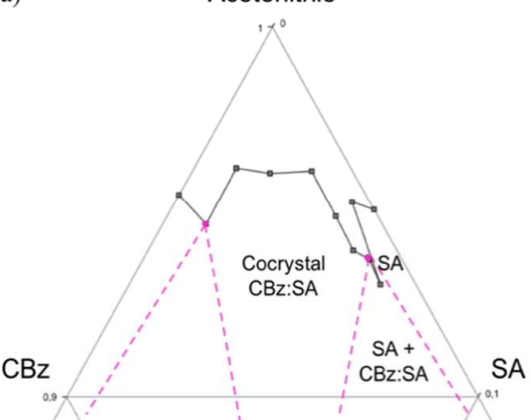

Figure S.3 - CA/SA ternary phase diagram at $9^{\circ} \mathrm{C}$ in $\mathrm{ACN}$. 
To crystallize the phase mixture, one has to be in the zone where both phases occur. This means that cooling a solution containing $10 \mathrm{~g} / \mathrm{l}$ of $\mathrm{CBZ}$ and $70 \mathrm{~g} / \mathrm{l}$ of SA (which is the concentration of the eutectic) under $9^{\circ} \mathrm{C}$ will lead to the crystallization of both CBZ:SA cocrystal and SA crystals.

\section{$\mathrm{CBZ} / \mathrm{SA}$ in $\mathrm{ACN}$ at $-10^{\circ} \mathrm{C}$}

Table S.3 Composition of initial vials with different ratios of CBZ and CA.

\begin{tabular}{|c|c|c|c|}
\hline Vial & W CBZ (mg) & $\begin{array}{c}\text { W SA } \\
(\mathrm{mg})\end{array}$ & $\begin{array}{c}\text { Eq SA } \\
\text { start }\end{array}$ \\
\hline 1 & 0,00 & 44,46 & $/$ \\
\hline 2 & 5,27 & 40,09 & 8,98 \\
\hline 3 & 7,57 & 35,21 & 1,89 \\
\hline 4 & 11,65 & 30,43 & 5,49 \\
\hline 5 & 16,47 & 26,33 & 3,08 \\
\hline 6 & 19,76 & 21,62 & 1,29 \\
\hline 7 & 24,62 & 17,87 & 0,86 \\
\hline 8 & 27,16 & 13,73 & 0,60 \\
\hline 9 & 32,37 & 9,16 & 0,33 \\
\hline 10 & 35,69 & 4,05 & 0,13 \\
\hline 11 & 42,46 & 0,00 & 0,00 \\
\hline
\end{tabular}

\begin{tabular}{|c|c|}
\hline $\begin{array}{c}\text { Eq SA } \\
\text { crystal }\end{array}$ & $\begin{array}{c}\text { Solid phase } \\
\text { (XRPD) }\end{array}$ \\
\hline 751,66 & CA \\
\hline 918,93 & CA \\
\hline 495,28 & CA \\
\hline 564,70 & CA \\
\hline 0,81 & CBZ:CA \\
\hline 0,76 & CBZ:CA \\
\hline 0,76 & CBZ:CA \\
\hline No Data & CBZ + \\
\hline 0,00 & CBZ:CA \\
\hline 0,00 & CBZ \\
\hline 0,00 & CBZ \\
\hline
\end{tabular}

Table S. 4 Composition of the supernatant solution after thermodynamic equilibrium has been reached.

\begin{tabular}{|c|c|c|c|c||c|c|c||c|}
\hline & \multicolumn{3}{|c||}{ Saturated solution composition } & \multicolumn{3}{c||}{ Mass percentage } & \multirow{2}{*}{$\begin{array}{c}\text { Solid } \\
\text { Phase }\end{array}$} \\
\cline { 2 - 7 } & $\begin{array}{c}\mathrm{CCBZ} \\
(\mathrm{mmol} / \mathrm{ml})\end{array}$ & $\begin{array}{c}\mathrm{CCA} \\
(\mathrm{mmol} / \mathrm{ml})\end{array}$ & $\begin{array}{c}\mathrm{n} \mathrm{ACN} \\
(\mathrm{mmol} / \mathrm{ml})\end{array}$ & Eq CA & $\% \mathrm{CBz}$ & $\% \mathrm{CA}$ & $\% \mathrm{ACN}$ & \\
\hline 1 & 0,000 & 0,222 & 19,15 & $/$ & 0,00 & 1,73 & 98,27 & $\mathrm{CA}$ \\
\hline 2 & 0,022 & 0,200 & 19,15 & 8,98 & 0,46 & 1,66 & 97,89 & CA \\
\hline 3 & 0,070 & 0,131 & 19,15 & 1,89 & 1,81 & 2,21 & 95,98 & CA \\
\hline 5 & 0,049 & 0,152 & 19,15 & 3,08 & 2,98 & 2,82 & 94,20 & CBZ:CA \\
\hline 6 & 0,084 & 0,108 & 19,15 & 1,29 & 2,20 & 1,49 & 96,30 & CBZ:CA \\
\hline 7 & 0,104 & 0,089 & 19,15 & 0,86 & 3,36 & 1,21 & 95,42 & CBZ:CA \\
\hline 8 & 0,115 & 0,069 & 19,15 & 0,60 & 5,41 & 1,54 & 93,05 & CBZ + \\
\hline 9 & 0,137 & 0,046 & 19,15 & 0,33 & 3,93 & 0,26 & 95,80 & CBZ \\
\hline 10 & 0,151 & 0,020 & 19,15 & 0,13 & 4,55 & 0,07 & 95,37 & CBZ \\
\hline 11 & 0,180 & 0,000 & 19,15 & 0,00 & 7,00 & 0,00 & 93,00 & CBZ \\
\hline
\end{tabular}

b)

Acetonitrile

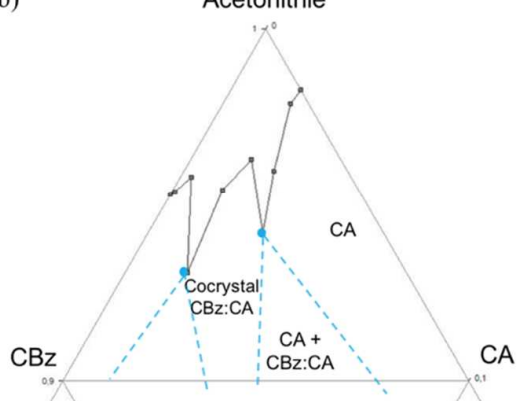

Figure S.4-CBZ/CA ternary phase diagram at $-10^{\circ} \mathrm{C}$ in $\mathrm{ACN}$. 
To crystallize a phase mixture, a solution of $17 \mathrm{~g} / \mathrm{l}$ of CBZ and $27 \mathrm{~g} / \mathrm{l} \mathrm{CA}$, was created. After dissolution at higher temperature, the solution was cooled to $-10^{\circ} \mathrm{C}$, and $1 / 3$ of the solution evaporated to increase the yield. This led to obtention of a crystallized mixture of cocrystal and CA.

\section{Paramagnetic solutions}

The paramagnetic solution composition was chosen in order to maximise the levitation height difference between compounds.

\section{Paramagnetic solution composition for CBZ and SA}

The levitation height difference was measured for six solutions of different composition and the density beads (beads whose density is known very precisely) of respectively $1.35 \mathrm{~g} / \mathrm{cm}^{3}$ and 1.45 $\mathrm{g} / \mathrm{cm}^{3}$ were used to represent CBZ:SA cocrystals $\left(1.356 \mathrm{~g} / \mathrm{cm}^{3}\right)$ and SA crystals $\left(1.443 \mathrm{~g} / \mathrm{cm}^{3}\right)$.

Table S. 5 Paramagnetic solutions tested for CBZ/SA.

\begin{tabular}{|c|c|c|c|c|c|}
\hline Solution & $\begin{array}{c}{\left[\mathrm{MnCl}_{2}\right]} \\
\mathrm{mol} / \mathrm{L}\end{array}$ & $\begin{array}{c}{[\mathrm{NaCl}]} \\
\mathrm{mol} / \mathrm{L}\end{array}$ & $\begin{array}{c}\text { Sucrose } \\
\mathrm{mol} / \mathrm{L}\end{array}$ & $\begin{array}{c}\text { Height } \\
\text { difference }(\mathrm{cm})\end{array}$ & $\begin{array}{c}\text { Both beads in } \\
\text { levitation? }\end{array}$ \\
\hline 1 & 2 & 1,2 & 0,2 & 1,2 & Yes \\
\hline 2 & 2 & 1,2 & 0,3 & 1,5 & Yes \\
\hline 3 & 2 & 1,2 & 0,5 & 1,6 & Yes \\
\hline 4 & 2 & 1,5 & 0,2 & 1,4 & Yes \\
\hline 5 & 2 & 1,5 & 0,3 & 1,4 & Yes \\
\hline 6 & 2 & 1,5 & 0,5 & $/$ & $\begin{array}{c}\text { Lighter bead } \\
\text { floating }\end{array}$ \\
\hline
\end{tabular}

Solution 5 , led to the density beads levitating at respectively one third and two thirds of the solution. It was chosen as the standard paramagnetic solution for the separation of CBZ:SA cocrystals from SA crystals. By weighing precisely $100 \mathrm{ml}$ of solution, we determined its density at $1.304 \mathrm{~g} / \mathrm{cm}^{3}$.

\section{Paramagnetic solution composition for CBZ and CA}

Density beads, were used of respectively $1,25 \mathrm{~g} / \mathrm{cm}^{3}$ to represent CBZ:CA crystals $\left(1.219 \mathrm{~g} / \mathrm{cm}^{3}\right)$ and $1.30 \mathrm{~g} / \mathrm{cm}^{3}$ to represent CA crystals $\left(1,257 \mathrm{~g} / \mathrm{cm}^{3}\right)$. As the densities of the beads are over-estimating the real densities of the crystals, we will be looking for levitation heights between one fifth and one quarter of the total height for the lower bead and between two fifth and one half for the higher one.

Table S. 6 Paramagnetic solutions tested for CBZ/SA.

\begin{tabular}{|c|c|c|c|c|c|}
\hline Solution & $\begin{array}{c}{\left[\mathrm{MnCl}_{2}\right]} \\
\mathrm{mol} / \mathrm{L}\end{array}$ & $\begin{array}{c}{[\mathrm{NaCl}]} \\
\mathrm{mol} / \mathrm{L}\end{array}$ & $\begin{array}{c}\text { [Sucrose] } \\
\mathrm{mol} / \mathrm{L}\end{array}$ & $\begin{array}{c}\text { Height } \\
\text { difference } \\
(\mathrm{cm})\end{array}$ & $\begin{array}{c}\text { Both beads in } \\
\text { levitation? }\end{array}$ \\
\hline 1 & 2 & 1,2 & 0,2 & $/$ & $\begin{array}{c}\text { Lighter bead } \\
\text { floating }\end{array}$ \\
\hline 2 & 1,5 & 1,5 & 0,3 & 0,5 & Yes \\
\hline 3 & 1,5 & 1 & 0,2 & 0,5 & Yes \\
\hline 4 & 1,2 & 1,5 & 0,3 & 0,7 & Yes \\
\hline 5 & 1,1 & 1,2 & 0,2 & 0,7 & Yes \\
\hline 6 & 1 & 1,5 & 0,2 & $/$ & Both sinking \\
\hline 7 & 1 & 1 & 0,2 & $/$ & Both sinking \\
\hline
\end{tabular}


In the context of this work, we decided to use solution 5 to separate CBZ:CA cocrystals from CA crystals because a smaller $\mathrm{MnCl}_{2}$ concentration should lead to a more sensitive separation (see Mirica, K. A., Shevkoplyas, S. S., Phillips, S. T., Gupta, M., \& Whitesides, G. M., Measuring densities of solids and liquids using magnetic levitation: Fundamentals. J. Am. Che. Soc., 2009, 131(29), 1004910058). 


\section{Physical mix separation}

For each system, we studied the quality of the MagLev separation for 16 samples: we tried to separate four different ratios of cocrystal and coformer crystal and repeated each experiment 4 times. This allowed us to verify the efficiency of the device under various conditions.

The four ratios we chose to test were:

1. $5 \mathrm{mg}$ cocrystals $+15 \mathrm{mg}$ coformer crystals

2. $8 \mathrm{mg}$ cocrystals $+13 \mathrm{mg}$ coformer crystals

3. $13 \mathrm{mg}$ cocrystals $+8 \mathrm{mg}$ coformer crystals

4. $15 \mathrm{mg}$ cocrystals $+5 \mathrm{mg}$ coformer crystals

In order to standardize the procedure, all cocrystal and coformer crystals were taken from the same batch of material, obtained from an acetonitrile crystallization process.

For both systems and all the ratios, a nice separation was observed in the MagLev, as illustrated in below.
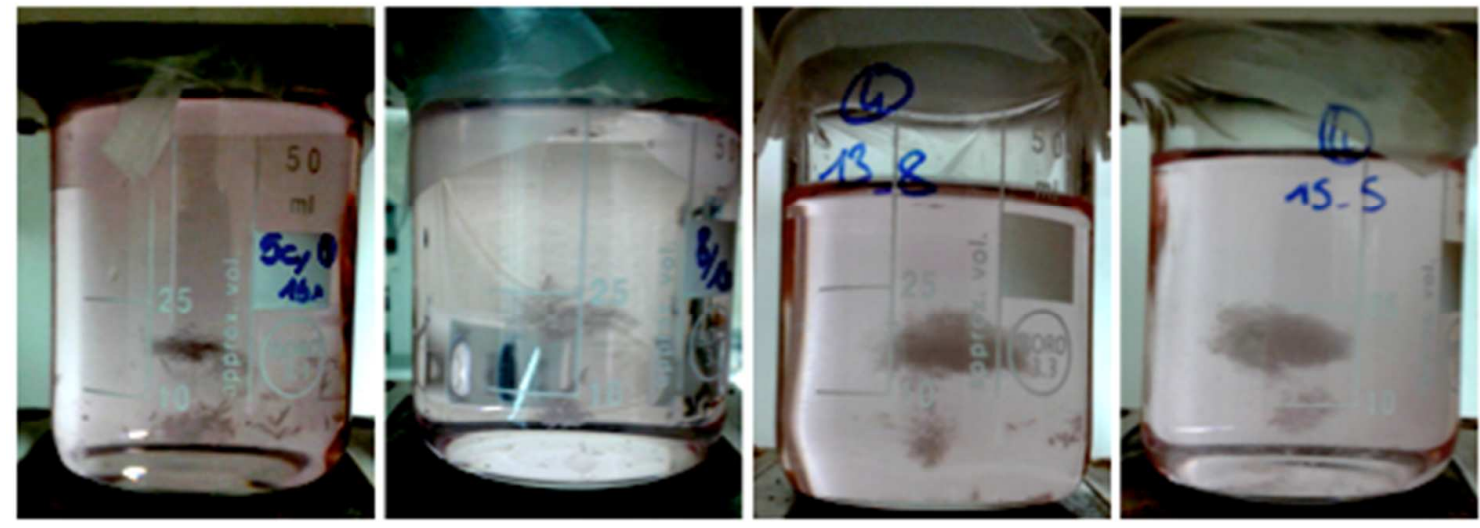

Figure S. 5 - Separation of a physical mix of CBZ:SA and SA crystals; from left to right $5+15 \mathrm{mg}, 8+13 \mathrm{mg}, 13+8 \mathrm{mg}$ and $15+5 \mathrm{mg}$
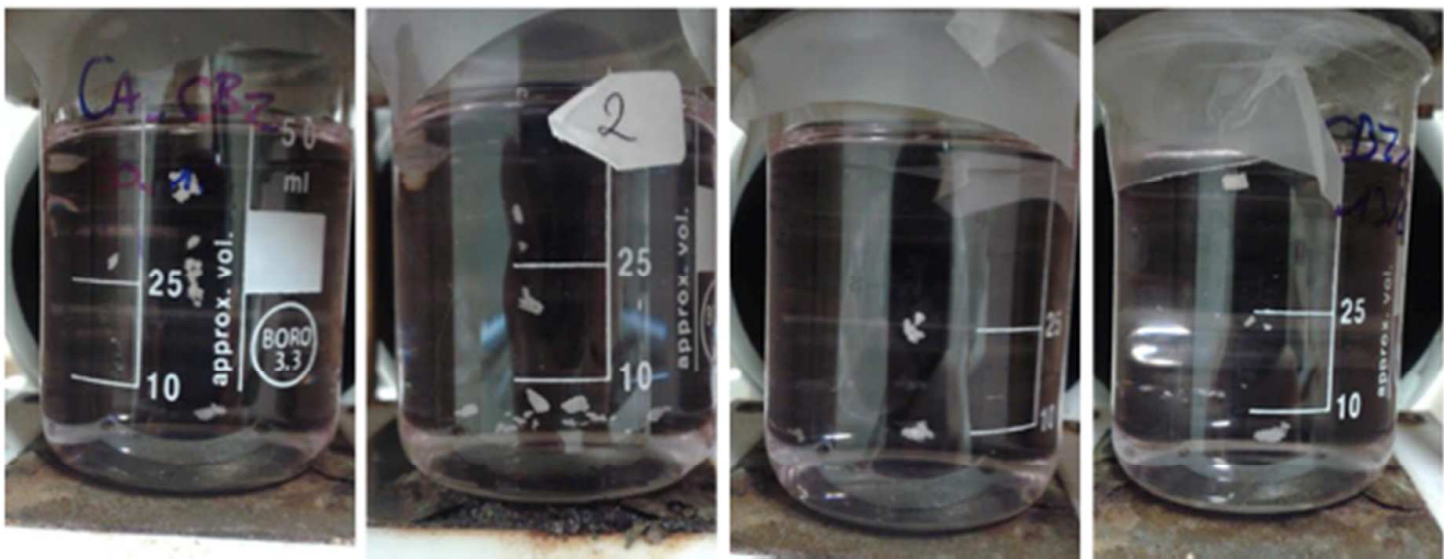

Figure S.6 - Separation of a physical mix of CBZ:CA ant CA crystals; from left to right $5+15 \mathrm{mg}, 8+13 \mathrm{mg}, 13+8 \mathrm{mg}$ and $15+5 \mathrm{mg}$

\section{Physical mix separation of CBZ:SA cocrystal and SA crystal}

The $95 \%$ precision interval was statistically deduced (based on the calibration curves).

Table S.7 Results on the separation of a physical mixture of CBZ :SA and SA crystals. 


\begin{tabular}{|c|c|c|c|c|c|c|c|}
\hline $\begin{array}{c}\text { W } \\
\text { Cocrystal } \\
(\mathrm{mg})\end{array}$ & $\begin{array}{c}\text { W } \\
\text { SA } \\
(\mathrm{mg})\end{array}$ & $\begin{array}{c}\text { Eq SA } \\
\text { Starting } \\
\text { Mix }\end{array}$ & $\begin{array}{c}\text { Eq SA } \\
\text { MagLev } \\
\text { Test } 1\end{array}$ & $\begin{array}{c}\text { Eq SA } \\
\text { Maglev } \\
\text { Test } 2\end{array}$ & $\begin{array}{c}\text { Eq SA } \\
\text { MagLev } \\
\text { Test } 3\end{array}$ & $\begin{array}{c}\text { Eq SA } \\
\text { MagLev } \\
\text { Test } 4\end{array}$ & \\
\hline \multirow{3}{*}{5} & \multirow{3}{*}{15} & \multirow{3}{*}{9,33} & 1,01 & 1,00 & 0,99 & 1,00 & Top cloud \\
\hline & & & {$[0,90-1,12]$} & {$[0,89-1,11]$} & {$[0,88-1,10]$} & {$[0,90-1,12]$} & $\begin{array}{c}\text { Top cloud } \\
95 \% \text { interval }\end{array}$ \\
\hline & & & 1100 & SA pur & 500 & SA pur & Bot. cloud \\
\hline \multirow{3}{*}{8} & \multirow{3}{*}{13} & \multirow{3}{*}{5,54} & 0,98 & 1,00 & 2,19 & 0,96 & Top cloud \\
\hline & & & {$[0,87-1,09]$} & {$[0,89-1,11]$} & {$[1,95-2,43]$} & {$[0,85-1,06]$} & $\begin{array}{c}\text { Top cloud } \\
95 \% \text { interval }\end{array}$ \\
\hline & & & 83 & 4700 & SA pur & SA pur & Bot. cloud \\
\hline \multirow{3}{*}{13} & \multirow{3}{*}{8} & \multirow{3}{*}{2,75} & 0,93 & 0,98 & 1,78 & 1,03 & Top cloud \\
\hline & & & {$[0,83-1,03]$} & {$[0,87-1,09]$} & {$[1,58-1,98]$} & {$[0,92-1,14]$} & $\begin{array}{c}\text { Top cloud } \\
95 \% \text { interval }\end{array}$ \\
\hline & & & 83 & 4700 & 2,97 & 12,6 & Bot. cloud \\
\hline \multirow{3}{*}{15} & \multirow{3}{*}{5} & \multirow{3}{*}{1,97} & 0,97 & 0,96 & 0,93 & 0,97 & Top cloud \\
\hline & & & {$[0,86-1,08]$} & {$[0,85-1,06]$} & {$[0,83-1,03]$} & {$[0,86-1,08]$} & $\begin{array}{c}\text { Top cloud } \\
95 \% \text { interval }\end{array}$ \\
\hline & & & No Data & No Data & 5,44 & SA pur & Bot. cloud \\
\hline
\end{tabular}

To determine if the starting composition of the mix has an influence on the purification, we used an ANOVA method. We already removed the two outliers to run this analysis.

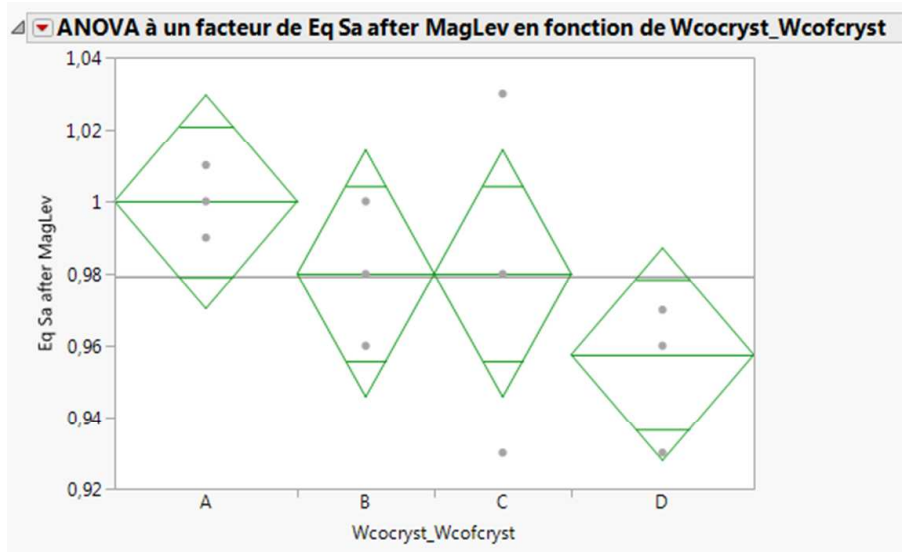

Figure S.7 - Anova analysis of system CBZ:SA and SA physical mix repeatability results

\section{Physical mix separation of CBZ:CA cocrystal and CA crystal}

Eq CA were measured using a calibration curve, as for the phase diagram measurements. We thus considered the same $11 \%$ error for a $95 \%$ precision interval.

Table S.8 Results on the separation of a physical mixture of CBZ :CA and CA crystals.

\begin{tabular}{|c|c|c|c|c|c|c|c|}
\hline $\begin{array}{c}\text { W } \\
\text { Cocrystal } \\
\text { (mg) }\end{array}$ & $\begin{array}{c}\text { W } \\
\text { CA } \\
\text { (mg) }\end{array}$ & $\begin{array}{c}\text { Eq CA } \\
\text { Starting } \\
\text { Mix }\end{array}$ & $\begin{array}{c}\text { Eq CA } \\
\text { MagLev } \\
\text { Test } 1\end{array}$ & $\begin{array}{c}\text { Eq CA } \\
\text { MagLev } \\
\text { Test } 2\end{array}$ & $\begin{array}{c}\text { Eq CA } \\
\text { MagLev } \\
\text { Test } 3\end{array}$ & $\begin{array}{c}\text { Eq CA } \\
\text { MagLev } \\
\text { Test } 4\end{array}$ & \\
\hline \multirow{3}{*}{5} & \multirow{3}{*}{15} & \multirow{3}{*}{9,33} & CA pur & CA pur & CA pur & CA pur & Top cloud \\
\hline & & & No Data & 0,79 & 0,97 & 1,00 & Bot. cloud \\
\hline & & & / & {$[0,70-0,88]$} & {$[0,86-1,08]$} & {$[0,89-1,11]$} & $\begin{array}{l}\text { Bot. cloud 95\% } \\
\text { interval }\end{array}$ \\
\hline \multirow{2}{*}{8} & \multirow{2}{*}{13} & \multirow{2}{*}{5,54} & CA pur & CA pur & 165,00 & 58,30 & Top cloud \\
\hline & & & 1,03 & 0,90 & 1,11 & 1,01 & Bot. cloud \\
\hline
\end{tabular}




\begin{tabular}{|c|c|c|c|c|c|c|c|}
\hline & & & {$[0,92-1,14]$} & {$[0,80-1,00]$} & {$[0,99-1,23]$} & {$[0,90-1,13]$} & $\begin{array}{c}\text { Bot. cloud 95\% } \\
\text { interval }\end{array}$ \\
\hline \multirow{3}{*}{13} & \multirow{3}{*}{8} & \multirow{3}{*}{2,75} & CA pur & CA pur & CA pur & CA pur & Top cloud \\
\hline & & & 1,01 & 1,02 & 1,01 & 0,99 & Bot. cloud \\
\hline & & & {$[0,90-1,13]$} & {$[0,91-1,13]$} & {$[0,90-1,13]$} & {$[0,88-1,10]$} & $\begin{array}{c}\text { Bot. cloud 95\% } \\
\text { interval }\end{array}$ \\
\hline \multirow{3}{*}{15} & \multirow{3}{*}{5} & \multirow{3}{*}{1,97} & 1649 & 426 & 187 & CA pur & Top cloud \\
\hline & & & 1,03 & 1,03 & 1,02 & 0,87 & Bot. cloud \\
\hline & & & {$[0,92-1,14]$} & {$[0,92-1,14]$} & {$[0,91-1,13]$} & {$[0,77-0,97]$} & $\begin{array}{l}\text { Bot. cloud 95\% } \\
\text { interval }\end{array}$ \\
\hline
\end{tabular}

As for the first system, we can try to determine if the starting composition of the mix has an influence on the purification using an ANOVA method (see fig. S.8). We already removed the outliers to run this analysis.

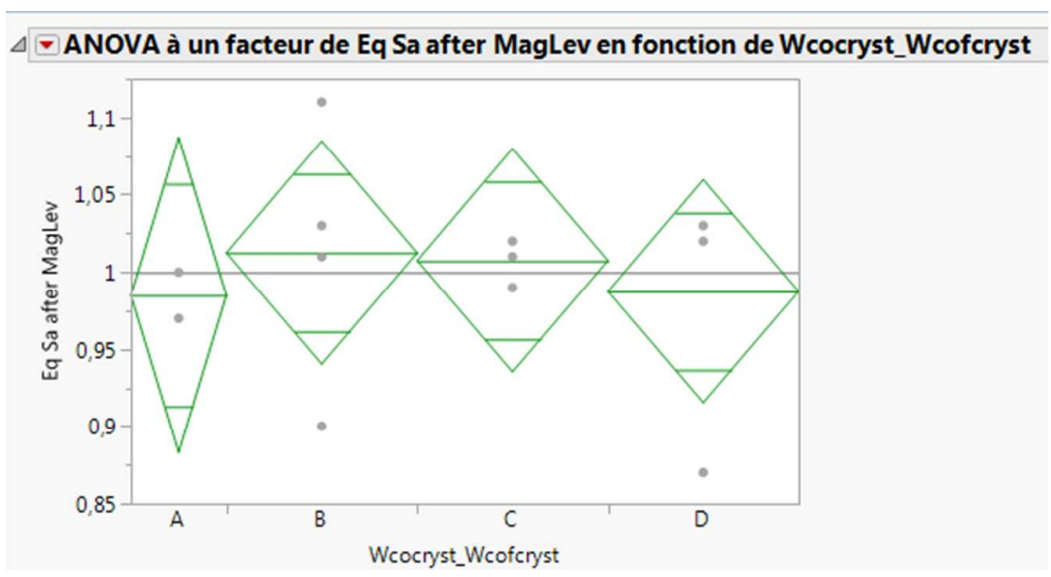

Figure S.8 - Anova analysis of system 2 physical mix repeatability results

The different categories of the Wcocryst_Wcofcryst parameter correspond from $A$ to $D$ to a mixture of $5 \mathrm{mg}$ cocrystals $+15 \mathrm{mg}$ SA crystals, $8+13 \mathrm{mg}, 13+8 \mathrm{mg}$ and $15+5 \mathrm{mg}$.

The variance of the means (mean square 0.000642) is way inferior to the variance of the results (mean square $0.0004188 ; \mathrm{F}$ ratio 0.1532 ). This means that the quality of the purification does not depend on the initial composition of the mixture. 


\section{Crystallized mix separation}

Crystallized mix separation of CBZ:SA cocrystal and SA crystal

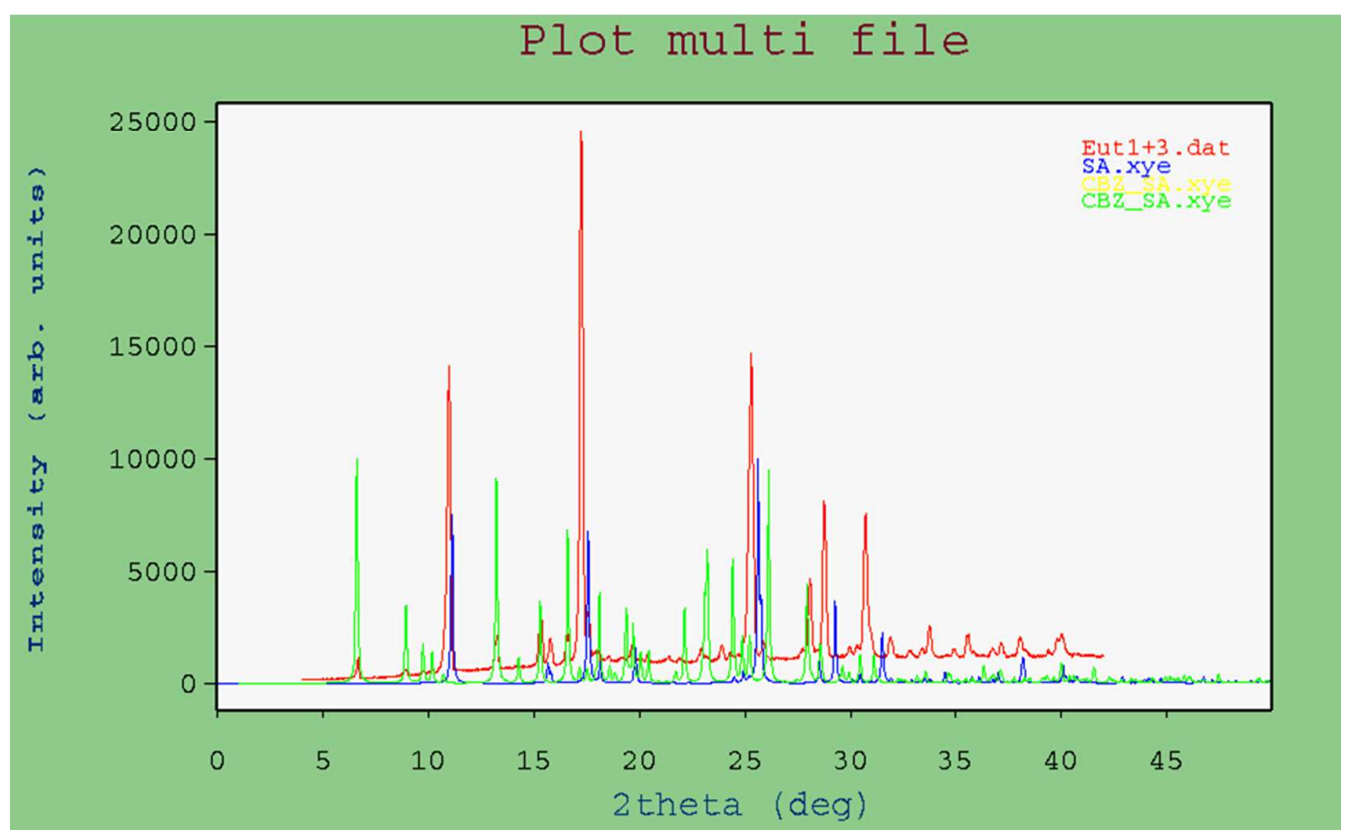

Figure S.9 - XRPD profile of System 1 eutectic (in red) compare to references SA (in blue) and CBZ:SA (in green) 